\section{The open question in selenology}

\author{
David W. Hughes
}

Origin of the Moon. Edited by W. K Hartmann, R. J. Phillips and G. J. Taylor. Lunar \& Planetary Institute, 3303 NASA Road 1, Houston, Texas 77058:1986. Pp.781.\$25.

EARTH's satellite Moon has a mass of 0.012453 Earth masses, a diameter of 0.27252 Earth diameters and, at present, is about $384,401 \mathrm{~km}$ away, this distance increasing by $4 \mathrm{~cm}$ per year. It has been assiduously observed throughout civilization, culminating in the period between July 1969 and December 1972 when 12 men walked on its surface and about 382 $\mathrm{kg}$ of rock was returned for analysis on Earth. In astronomical terms, therefore, the Moon must be classed as a well-known object, but astronomers still have to admit shamefacedly that they have little idea as to where it came from. This is particularly embarrassing, because the solution of the mystery was billed as one of the main goals of the US lunar exploration programme.

Science is replete with "if onlys". In the lunar case things might have been different if only some primordial "genesis" rock, $4.5 \times 10^{9}$ years old, had been found; chemical, isotopic and petrologic analysis of the rock might have provided the necessary clues. Astronauts were trained to look for it, but only a few chips were found. The extraordinarily intense lunar bombardment, which was associated with the sweeping up of planetesimal debris and which occurred between $4.5 \times 10^{9}$ and $4.0 \times 10^{9}$ years ago, blanketed the Moon with a pulverized regolith and hid the evidence.

Twelve years after the last lunar landing was as good a time as any to hold a conference on the subject of selenology. The conference was organized by the Lunar and Planetary Sample Team and took place in Kona, Hawaii, in October 1984. Thirty three papers from the meeting are published in the volume under review, and in one way the reviewer's job is easy - the book cannot be compared with others on the subject because there are none.

As with most studies of origin mechanisms, the starting point must be the known facts. Moving beyond the mass, size and angular momentum, data that can easily be gleaned from Earth observation, a large number of important clues have come from sample analysis. Lunar rocks and soils are depleted in volatile elements, much more so than those on Earth, this loss probably being caused by some highly energetic process that heated lunar material to high temperatures. Iron has been depleted to a value of only 25 per cent of what one would expect from cosmic abundance. The oxygen isotopic ratio is similar to that of Earth. And comparing lunar material with rocks from the Earth's mantle it has been found that there are strong similarities between the relative abundances of the siderophile elements (such as $\mathrm{Co}, \mathrm{Ni}, \mathrm{W}, \mathrm{P}, \mathrm{S}, \mathrm{Se}, \mathrm{Te}$ ) and lithophile elements (V, Cr, Mn).

Plagioclase takes up to 75 per cent of lunar surface material and could only have been produced by the partial melting of a crustal layer several hundred kilometres thick followed by crystal fractionation. This again points to a high-energy origin process. If the celestial clock is wound back it is clear that $4.5 \times 10^{9}$ years ago the Moon was very much closer to the Earth - about 10 Earth radii away $(60,000 \mathrm{~km})$

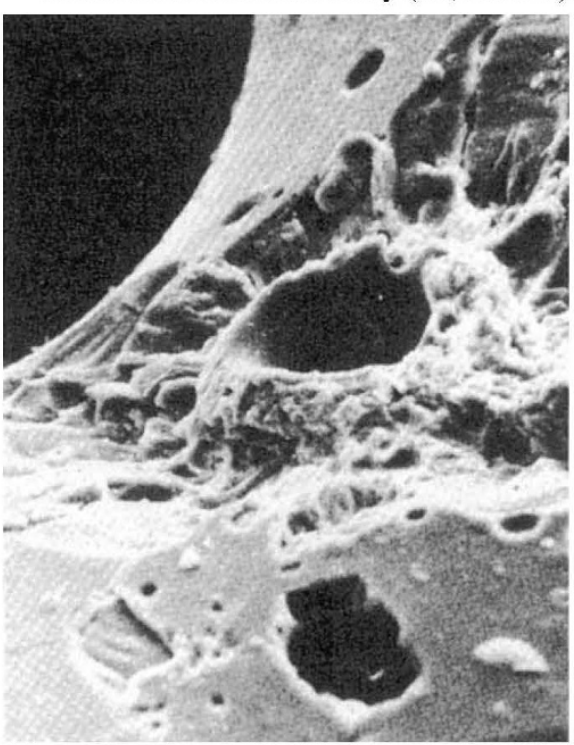

Zap crater, the result of the impact of a highspeed meteoritic dust grain on Moon rock. The picture is reproduced from Discovering the Universe, by William J. Kaufmann III, a textbook for a one-term, descriptive course on astronomy. Publisher is W. H. Freeman, price is $p b k \$ 24.95, £ 24.95$.

- with a direct orbit inclined at $10^{\circ}$ to the Earth's equatorial plane. About 400 pages and 16 papers in the book are taken up by a discussion of the dynamical, geochemical, petrologic, isotopic, thermal and magnetic facts relating to the EarthMoon system. The remainder covers ideas about lunar origin.

Five theories are studied in detail. According to the first of them, "intact capture", the Moon was formed somewhere else in the Solar System and originally had a heliocentric orbit. It was then captured intact by the Earth. Originally it was thought that this theory could account for a very exotic lunar composition. Unfortunately the capture process is highly dependent on velocity, requiring a very low relative velocity between Earth and Moon. This occurs only if the Moon's original orbit had a radius between 0.95 and 1.05 AU - just like that of Earth, so they would probably have been formed out of the same material anyway.

The second theory, "co-accretion", holds that Earth and Moon formed at the same time, the Moon accreting from a disk or swarm of solid particles that were orbiting the Earth. Formation of the Moon would be like planetary formation, but on a different scale. Unfortunately, in this model there are difficulties in explaining the differences in composition, providing the energy to melt the magma and supplying the angular momentum. Collisions between particles in the disk could, however, have led to devolatilization.

"Earth fission", the third theory, was first proposed by G. H. Darwin in 1879 . He thought that free oscillations in a fastspinning ( $\sim 4$ hour $)$ proto-Earth would be influenced by solar tides, and would produce a tidal bulge which grew higher and higher until the tip of a highly elongated proto-Earth broke off to form the Moon. Modern versions invoke rotational instability in a proto-Earth which had a 2.6hour day. Core segregation caused the spin rate to increase, making the shape distort until the more tenuously connected end broke off. The Moon probably did not break off intact. According to a more likely model, dispersed material was shed from the elongated Earth, this material forming a disk which subsequently condensed to form the Moon. The main problem with this idea is that the Earth-Moon system needs four times more angular momentum than it has at present.

The fourth theory is "collision ejection", which has the Earth being hit by a planetesimal of about 0.1 Earth masses. A large expanding cloud of vapour and dust was produced by this collision. Some fell back to Earth, some escaped into space, but enough of it went into orbit eventually to accrete to form the Moon.

Finally there is "distintegrative capture", in which a loosely bound planetesimal passed close enough to Earth to enter the Roche limit, was tidally disrupted and part of the debris was subsequently captured and condensed to form the Moon.

All of these theories are reviewed in detail in Origin of the Moon. This is a fascinating subject, and it has been well served by both authors and editors - the book is well produced, thorough, carefully structured, up to date and well referenced. It shows that scientific fashion wanders from theory to theory, all of which have good and bad points. At the moment co-acccretion is retreating from favour and collision ejection is in the ascendent. But astronomy would be rather dull if we knew all the answers and it is clear from this book that "Where did the Moon come from?" is still an open, exciting and challenging question.

David W. Hughes is Senior Lecturer in Astronomy and Physics, Department of Physics, University of Sheffield, Sheffield S3 $7 R H, U K$. 